\title{
Developmental Patterning of Rod and Cone Photoreceptors in Embryonic Zebrafish
}

\author{
PAMELA A. RAYMOND, LINDA K. BARTHEL, AND GARY A. CURRAN \\ Department of Anatomy and Cell Biology, University of Michigan Medical School, \\ Ann Arbor, Michigan 48109-0616
}

\begin{abstract}
Cone photoreceptors in the zebrafish retina are arranged in a crystalline lattice, with each spectral subtype at a specific position in the array; rod photoreceptors are inserted around the cones. Patterning events and developmental mechanisms that lead to the formation of the cone mosaic are not known. To begin investigating this issue, we examined the initial stages of opsin expression in zebrafish embryos by in situ hybridization with goldfish opsin cRNA probes to determine how and when the cone mosaic pattern arises. We found both differences and similarities in the spatiotemporal patterns of rod and cone development, which suggest the following: 1) Expression of opsin message (including rod opsin, blue and red cone opsins) was initiated at $50-52$ hours postfertilization by a few photoreceptors which were consistently found in a ventral patch of retina located nasal to the choroid fissure. 2) The cone mosaic pattern was generated by a crystallization-like process initiated in the precocial ventral patch and secondarily in nasal retina, which then swept like a wave into dorsotemporal retina. 3) The pattern of differentiation of rods in the ventronasal patch differed substantially from that in the remainder of the retina, suggesting that these precocial rods might differ from typical rods. 4) Developmental maturation of rods in zebrafish, as reflected by expression of opsin, may be accelerated compared to cones, which are thought to become postmitotic before rods. These data are consistent with a model in which lateral inductive interactions among differentiating photoreceptors lead to patterning of the array. 1995 Wiley-Liss, Inc.
\end{abstract}

Indexing terms: retina, opsin, in situ hybridization, Cyprinidae

Two fundamental organizational principles of the vertebrate retina are that major categories of neurons are stratified, like the sheets in a layer cake, and that within a given stratum, subtypes of neurons are distributed with a regular spacing, typically abutting but often not overlapping one another, like the tiles on a floor (Wässle and Reimann, 1978; Marc, 1986). In some vertebrate species, most notably in teleost fish, the mosaic arrangement of cone photoreceptors is especially striking-teleost cones are typically arrayed in a two-dimensional, crystalline lattice of highly regular and repeating mosaic units, with the different spectral/morphological subtypes of cones located at specific positions in the array (Engström, 1960, 1963; Raymond et al., 1993a). In mammals, including primates (Marc and Sperling, 1977; de Monasterio et al., 1981; Ahnelt et al., 1987; Wikler et al., 1990) and rodents (Szél et al., 1988), the cones sensitive to short wavelengths ("blue" cones) are arranged in a regular lattice pattern, but other spectral classes (those sensitive to long or medium wavelengths, i.e., "red" and "green" cones) although not randomly distributed, are not as regularly spaced as the blue cones. In both fish and mammals, rod photoreceptors fill in the spaces between cones, but do not themselves form an independent mosaic pattern.

Photoreceptor density is typically not constant across the retina, and these topological variations in planimetric density (i.e., number of cells per unit retinal surface area) are reflected in the repeat distance of the cone mosaic unit, and sometimes in its composition. In zebrafish, the subject of this report, the density of rods is about twofold greater in ventral than in dorsal retina (Nawrocki, 1985), whereas the reverse is true for cones (P.A. Raymond and S.-Y. Lin, unpublished observations). The pattern and composition of the cone mosaic remains constant throughout the zebrafish retina (Engström, 1960). There are four morphological subtypes of cones in zebrafish: double cone pairs (each with a principal and an accessory member) and long single and

Accepted January 26, 1995.

Address reprint requests to Dr. Pamela A. Raymond, Dept. of Anatomy and Cell Biology, University of Michigan Medical School, Ann Arbor, MI 48109-0616. 
short single cones (Engström, 1960; Branchek and BreMiller, 1984; Nawrocki et al., 1985). Spectral identifications of morphological classes, based on both microspectrophotometry (Robinson et al., 1993) and in situ hybridization with cone opsin RNA probes (Raymond et al., 1993a; Robinson et al., 1993), indicate that the principal member of the double cone pair contains a long-wavelengthsensitive (red) pigment and the accessory member a middlewavelength-sensitive (green) pigment, the long single cone contains a short-wavelength-sensitive (blue) pigment, and the short single cone has an ultraviolet-sensitive pigment. The cone mosaic in zebrafish consists of alternating rows of double cones and single cones; along a row of double cones, the orientation of red and green is alternately reversed, and along a row of single cones, blue and ultraviolet cones alternate (Engström, 1960; Larison and BreMiller, 1990; Raymond et al., 1993a; Robinson et al., 1993). These rows are orthogonal to the retinal margin and radiate from the center of the retina like spokes on a wheel. Rods are interspersed among the cones, and continue to accumulate as the fish grows, as a result of the continued proliferation of rod precursors in the outer nuclear layer (Raymond, 1985a; Fernald, 1991).

The developmental mechanisms that control cell fate specification, that regulate cell density, and that produce the highly organized mosaics of photoreceptors and other neurons in the vertebrate retina are not well understood (Altshuler et al., 1991; Raymond, 1991; Reh, 1992; Adler, 1993). This process may involve a series of progressively more restrictive developmental decisions, such as, first, the choice of whether to become a ganglion cell or a photoreceptor, and if a photoreceptor, whether to be a rod or a cone, and if a cone, what spectral subclass to become (Harris and Messersmith, 1992). To produce the orderly patterns characteristic of cone mosaics, it seems essential that the developing photoreceptor cells communicate with their neighbors, sharing information about position and identity. Cell-cell communication involving inductive interactions that control commitment and choice of cell identity has been demonstrated for photoreceptor cells in the ommatidia of the adult compound eye of Drosophila, and in some cases, the relevant signaling molecules and signal transduction components have been identified and cloned (Cagan and Zipursky, 1992). Since the photoreceptors in the compound eye of the fly are arranged in a highly regular, crystalline lattice, in some ways reminiscent of the vertebrate cone mosaic, it is intriguing to speculate that similar developmental mechanisms might control pattern formation in both cases (Wikler and Rakic, 1991; Raymond, 1995).

How the teleost cone mosaic becomes organized during retinal development has been a subject of continuing interest for many years (Müller, 1952; Lyall, 1957; Ahlbert, 1973; Kunz et al., 1983; Larison and BreMiller, 1990). More recently, developmental studies of the cone mosaic in mammals have also appeared (Wikler and Rakic, 1991; Szél et al., 1993; Bumsted et al., 1994; Wikler and Rakic, 1994). These recent studies have been stimulated by the availability of antibodies that selectively recognize different cone opsins, thus allowing the spectral classes of cones to be identified as soon as opsin expression begins and before overt morphological differences appear. With the cloning of rod and cone opsin genes from many different vertebrate species, including goldfish (Johnson et al., 1993) and zebrafish (Robinson et al., 1993; Reece et al., 1994), together with the development of colorimetric in situ hybridization methods that allow cellular, and even subcellular, resolution of opsin messages (Barthel and Raymond, 1993; Raymond et al., 1993a), it has become possible to examine presumptive photoreceptors when they become committed to a particular spectral class and begin to express a specific opsin. The objective of the present study was to examine the initial steps in the formation of the cone mosaic pattern, and to compare the patterns of development of rods and cones in order to define reasonable mechanistic hypotheses for further investigations. Preliminary accounts of this work have appeared in abstract form (Raymond et al., 1993b; Raymond and Barthel, 1994).

\section{MATERIALS AND METHODS Tissue preparation for standard histology}

Adult zebrafish (Brachydanio rerio), $2.5-4.0 \mathrm{~cm}$ total length, were obtained from a local pet store and maintained on a 14-hour light:10-hour dark cycle in $0.5 \mathrm{~g} /$ liter Instant Ocean (Aquarium Systems, Mentor, $\mathrm{OH}$ ) at $28.5^{\circ} \mathrm{C}$. Breeding was according to published procedures (Westerfield, 1993).

Zebrafish embryos were collected approximately 2 hours postfertilization (PF) and maintained in 4-liter plastic trays at $28.5^{\circ} \mathrm{C}$. Fertilization was assumed to occur within 15 minutes of light onset, and the developmental age is given as hours PF. Since pigmentation in the wild-type zebrafish eye interferes with the visualization of cellular details in the whole mounts, to block its formation, embryos were placed in $0.2 \mathrm{mM}$ 1-phenyl-2-thiourea (PTU) at 12 hours PF (Westerfield, 1993). Embryos treated with PTU from 12 hours PF, and then removed from PTU at 4 days PF appeared to develop normally to adulthood with only slightly diminished skin pigmentation.

For standard light microscopic observations, adult fish were anesthetized in $0.02 \%$ methane tricaine sulfonate (Sigma) and then decapitated. The eyes were enucleated, fixed in $4 \%$ paraformaldehyde, $0.1 \%$ glutaraldehyde, $0.2 \%$ picric acid, and $5 \%$ sucrose in $0.1 \mathrm{M}$ phosphate buffer, $\mathrm{pH}$ 7.4 , for 1 hour at room temperature, then rinsed, dehydrated in graded ethanols, and embedded in glycol methacrylate (BioRad Polaron Instruments). Embryos were dechorionated with forceps, fixed, and processed similarly Sections were cut at $3 \mu \mathrm{m}$ and stained with Lee's stain (Bennett et al., 1976).

\section{Immunocytochemistry}

Adult eyes or whole embryos were fixed in $4 \%$ paraformaldehyde for 1 hour at room temperature, rinsed in phosphate buffer with $5 \%$ sucrose, and used for whole-mount immunocytochemistry or prepared for 3- $\mu \mathrm{m}$ cryosections as previously described (Barthel and Raymond, 1990).

A complete description of the protocol for immunocytochemistry on cryosections has been published (Barthel and Raymond, 1990). FRet43 (a gift from R. BreMiller) is a monoclonal antibody generated from a zebrafish retinal preparation; it labels an uncharacterized plasma membrane epitope on double cone photoreceptors, both red and green members of the pair (Larison and BreMiller, 1990). FRet43 was supplied as hybridoma culture supernatant, and was diluted 1:100. ROS1 is a monoclonal antibody generated from a homogenate of goldfish photoreceptor outer segments; it labels an uncharacterized epitope on rod outer 
segments in both goldfish and zebrafish (Raymond et al., 1993a). ROS1 was used as ascites fluid diluted 1:1,000. The monoclonal antibodies were detected by indirect immunofluorescence with fluorescein isothiocyanate (FITC)-conjugated secondary antibody (Sigma). Sections were viewed with a Leitz Aristoplan epifluorescent microscope equipped with Nomarski interference optics.

Immunocytochemistry on whole embryos was performed essentially as previously described for adult goldfish retinal whole-mounts (Braisted and Raymond, 1993). To visualize cone photoreceptors, FRet43 was diluted 1:100; to visualize rod photoreceptors, ROS1 was diluted 1:500. The avidinbiotin-peroxidase method was used for immunoperoxidase detection of both antibodies. For indirect immunofluorescent detection of FRet43, FITC-conjugated anti-mouse IgG $\mathrm{F}\left(\mathrm{ab}^{\prime}\right)_{2}$ fragment (Sigma, St. Louis, MO) was used. Heads or isolated eyes were mounted, coverslipped, and viewed with Nomarski optics on a Leitz Aristoplan microscope or imaged on a BioRad MRC 600 laser scanning confocal imaging system equipped with a krypton/argon laser.

\section{In situ hybridization}

Methods for whole-mount in situ hybridization were based on published protocols (Hemmati-Brivanlou et al., 1990; Coutinho et al., 1992). Dechorionated embryos were fixed overnight at $4^{\circ} \mathrm{C}$ in $4 \%$ paraformaldehyde, $0.5 \%$ glutaraldehyde in $0.1 \mathrm{M}$ phosphate buffer, pH 7.4 (Uehara et al., 1993), or alternatively in $4 \%$ paraformaldehyde for 1 hour at room temperature. Fixed embryos were rinsed and dehydrated in either $70 \%$ ethanol or $100 \%$ methanol and stored at $-20^{\circ} \mathrm{C}$ in $70 \%$ ethanol or $100 \%$ methanol for at least 24 hours before further processing. After discovering that the mixed aldehyde fixative was preferable for the cone opsin probes (see Results), some embryos fixed in paraformaldehyde were subsequently rehydrated and postfixed in paraformaldehyde/glutaraldehyde as described above.

Goldfish opsin cDNA clones (a gift from K. Nakanishi; Johnson et al., 1993) GFrod, GFred, and GFblu (rod, red cone, and blue cone opsins, respectively), subcloned in pBluescript II SK (Stratagene), were linearized and used to generate digoxigenin (DIG)-labeled RNA sense and antisense probes by in vitro transcription according to the manufacturer's specifications (Boehringer Mannheim) as described previously (Raymond et al., 1993a). In vitro transcription was carried out by linearizing the plasmids with either XbaI or HindIII restriction enzymes followed by transcription of the complete opsin coding sequences from either the T7 or T3 promoters to generate both sense and antisense DIG-labeled RNA probes. In some cases, the probes were treated by alkaline hydrolysis to reduce the length.

Embryos (stored at $-20^{\circ} \mathrm{C}$ ) were warmed to room temperature, dehydrated, cleared in xylene, and rehydrated in an alcohol series in phosphate buffered saline (PBS) with $0.1 \%$ Tween-20 (PBST). Those fixed in paraformaldehyde/ glutaraldehyde were treated with $30 \mu \mathrm{g} / \mathrm{ml}$ proteinase $\mathrm{K}$ (Boehringer Mannheim Biochemicals, Indianapolis, IN) in Tris buffer with ED'TA for $1-2$ hours at $37^{\circ} \mathrm{C}$, rinsed, and postfixed for 20 minutes in $4 \%$ paraformaldehyde. Embryos fixed in paraformaldehyde alone were treated with 10 $\mu \mathrm{g} / \mathrm{ml}$ proteinase $\mathrm{K}$ for 15 minutes at $37^{\circ} \mathrm{C}$. They were hybridized with $2-10 \mu \mathrm{g}$ probe $/ 500 \mu$ l hybridization solution at 55 to $60^{\circ} \mathrm{C}$ for $18-36$ hours with constant rotation.

Posthybridization procedures were carried out as described (Püschel et al., 1992; Barthel and Raymond, 1993).
Immunocytochemical detection of the DIG-labeled RNA probes was according to the manufacturer's instructions (Boehringer Mannheim) in 0.1\% Tween-20 with alkaline phosphate substrates 4-nitroblue tetrazolium chloride with 5-bromo-4-chloro-3-indolyl phosphate (NBT/BCI) or Vector Red (Vector Laboratories, Burlingame, CA); $1 \mathrm{mM}$ levamisol was added to block endogenous alkaline phosphatase activity (Westerfield, 1993). Heads or isolated eyes were mounted in $100 \%$ glycerol and viewed with Nomarski optics or, for the fluorescent Vector Red preparations, with the laser scanning confocal imaging system. Images were digitized on a Kodak PhotoCD (Eastman Kodak Co., Rochester, NY) and printed with Adobe Photoshop (Adobe Systems Inc., Mountain View, CA).

\section{RESULTS \\ Photoreceptors, both rods and cones, first differentiate in a localized ventral patch}

At about 2 days $P F$, rods and cones appeared in the embryonic zebrafish retinas on the nasal wall of the choroid fissure in ventral retina. This region was the first to laminate (Fig. 1A), indicating that retinal differentiation commences in ventral retina in zebrafish embryos. Immunocytochemistry with rod-specific (ROS-1; Fig. 2A) and doublecone-specific (FRet43; Fig. 2B,C) monoclonal antibodies on embryos at 50 hours PF revealed a small patch of less than five immunoreactive rods (Fig. 1C) and a similar number and distribution of presumptive double cones (Fig. 1D), tightly clustered in ventral retina.

By 3 days PF, the entire retina was laminated (Fig. 1B). It appeared, therefore, that a wave of differentiation had swept across the retina during this 24-hour period, leaving in its wake an organized retina with a discrete photoreceptor layer. Because the monoclonal antibodies we had used in these initial studies recognized uncharacterized epitopes with distinctly different subcellular distributions, it was not possible to make a careful comparison of the time course of development of rods and multiple subtypes of cones with these reagents. By examining opsin expression with in situ hybridization, we could study rod and cone development with approximately equivalent reagents and thereby compare their spatiotemporal patterns of differentiation.

\section{Precocial rods in the ventral patch accumulated rapidly and at high density, but elsewhere rods were added slowly and sporadically}

Rod opsin cRNA probes were visualized in wholemounted zebrafish embryos fixed either in paraformaldehyde alone or in paraformaldehyde/glutaraldehyde. Figure $3 \mathrm{~A}$ shows a whole-mount preparation of an embryo at 50 hours PF, viewed from the right side; in this embryo it appeared that a single rod in ventral retina expressed opsin. When viewed at a more superficial plane of focus, the choroid fissure could be seen (Fig. 3B), and it was apparent that the single-labeled rod lay adjacent and nasal to the choroid fissure. When this embryo was turned on its back and observed from the ventral side (Fig. 3A, inset), it again appeared that the right eye contained a single, heavily labeled rod, but now a second weakly labeled rod could be seen. In contrast, the left eye had four heavily labeled and perhaps two weakly labeled rods (data not shown).

Of 13 zebrafish eyes examined at 50 hours PF, nine eyes had 1 to 5 labeled rods and the other four eyes had none. In 


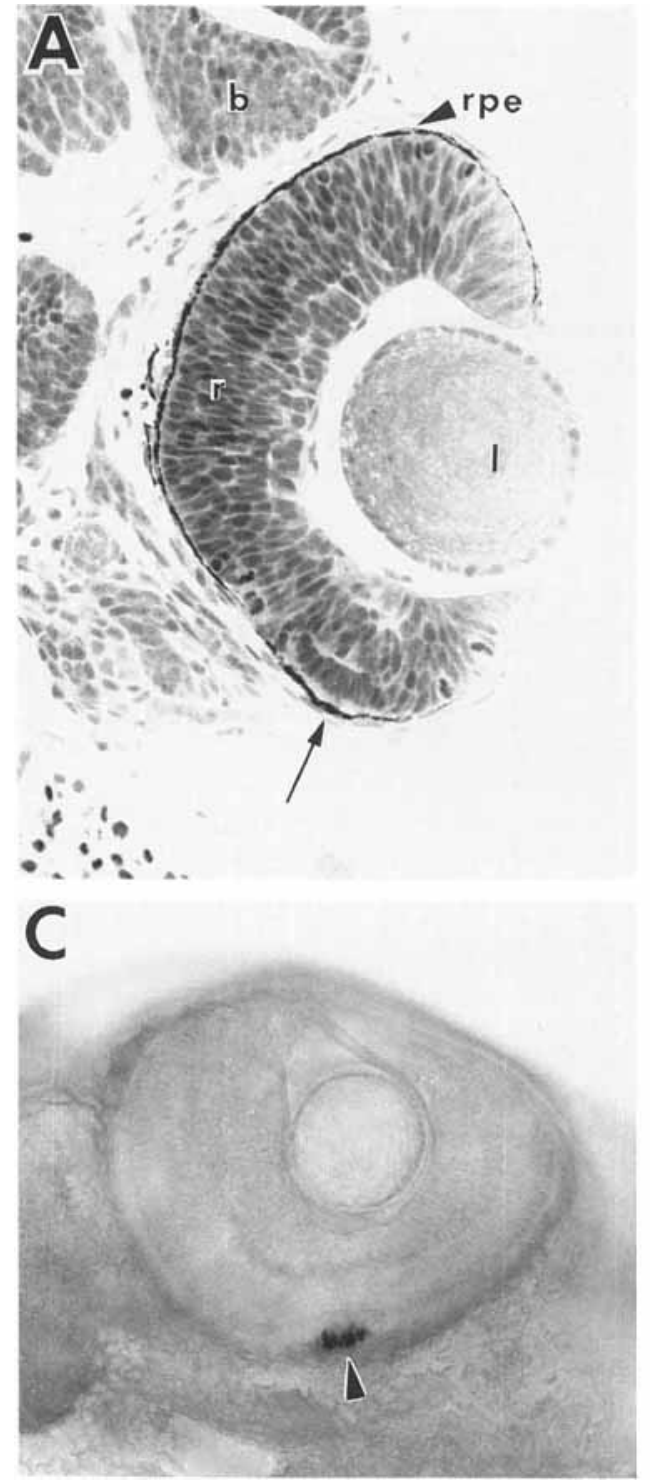

Fig. 1. A: Histological section through the head of a zebrafish embryo at 49 hours postfertilization (PF). Dorsal is up in all figures unless stated otherwise. The arrow indicates the region where retinal lamination has begun. $b$, brain; $r$, retina; 1 , lens; rpe, retinal pigmented epithelium. B: Histological section of a zebrafish embryo head at 73 hours PF. The arrow indicates the region where retinal lamination began. onl, outer nuclear layer; on, optic nerve; other abbreviations as in A. Bar $=50 \mu \mathrm{m}$; also applies to A. C: Whole-mounted zebrafish at 50

all cases, the labeled rods were in ventral retina, adjacent and nasal to the choroid fissure, and all labeled rods in a given eye were tightly clustered together. Therefore, we conclude that at 50 hours PF in zebrafish embryos, the first rod photoreceptors begin to express opsin message, and that these earliest rods are consistently located in a specific retinal location.

Over the next few hours, additional rods were added to the ventral patch, which thereby increased to approximately $30 \mu \mathrm{m}$ in diameter (Fig. 3C). Subsequently, a few isolated rods appeared outside the patch in sporadic locations (Fig. 3D). In order to quantitate this developmental progression in the differentiation of rods across the retina,
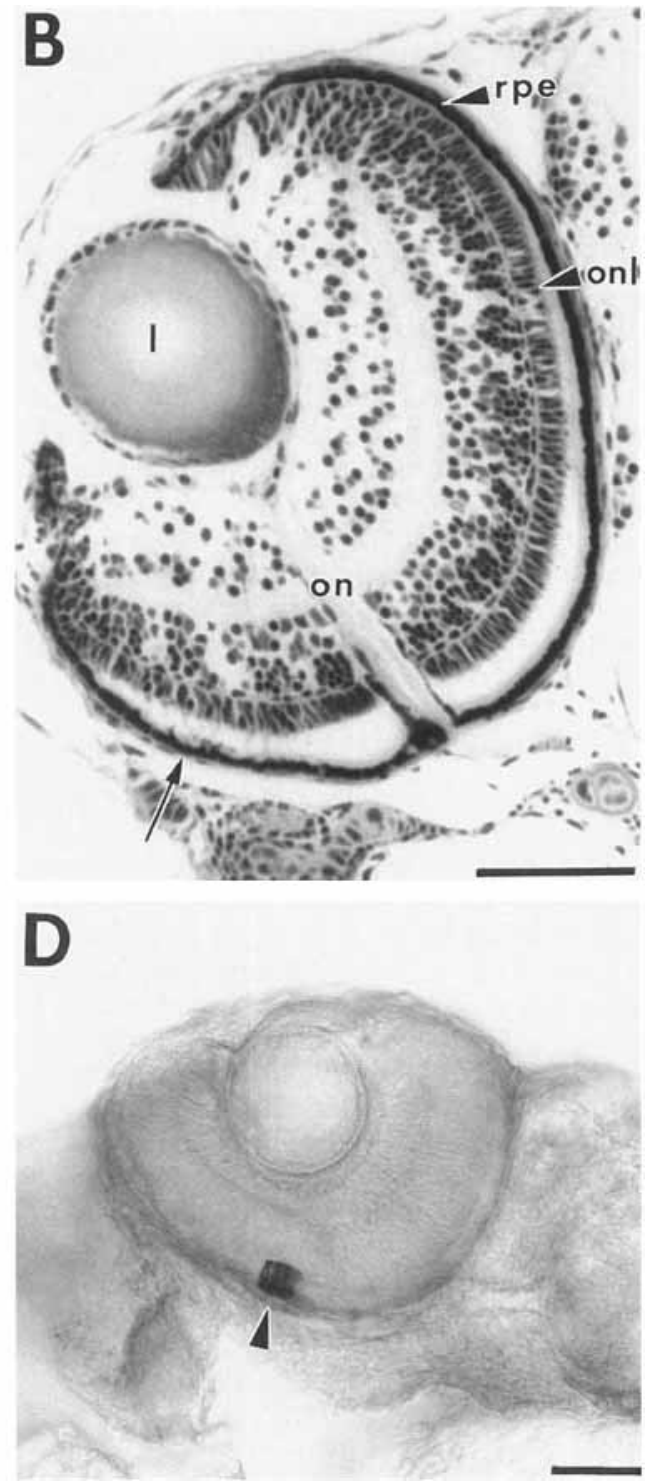

hours PF stained with ROS1 (immunoperoxidase). The view is from the ventral side of the embryo, and nasal is to the left. The arrowhead indicates a patch of immunofluorescent rod outer segments in ventral retina. Abbreviations as in A. D: Whole-mounted zebrafish at 50 hours PF stained with FRet43 (immunoperoxidase). Again the view is ventral, but nasal is to the right. The arrowhead indicates a patch of labeled double cones in ventral retina. Bar $=50 \mu \mathrm{m}$; also applies to $\mathrm{C}$.

we identified six stages of rod recruitment. Although the criteria used to distinguish stages are somewhat arbitrary, they were chosen for convenience, because they produced easily identifiable categories. The 6 stages are illustrated in the camera lucida drawings in Figure 4 and defined as follows:

Stage $0-$ No cells express rod opsin.

Stage 1-Fewer than 20 (and typically less than 10) opsin-expressing rods in a patch of ventral retina.

Stage 2-Ventral patch of greater than 20 rods.

Stage 3 -Ventral patch of rods too dense to count individual cells, scattered rods outside the patch, and 

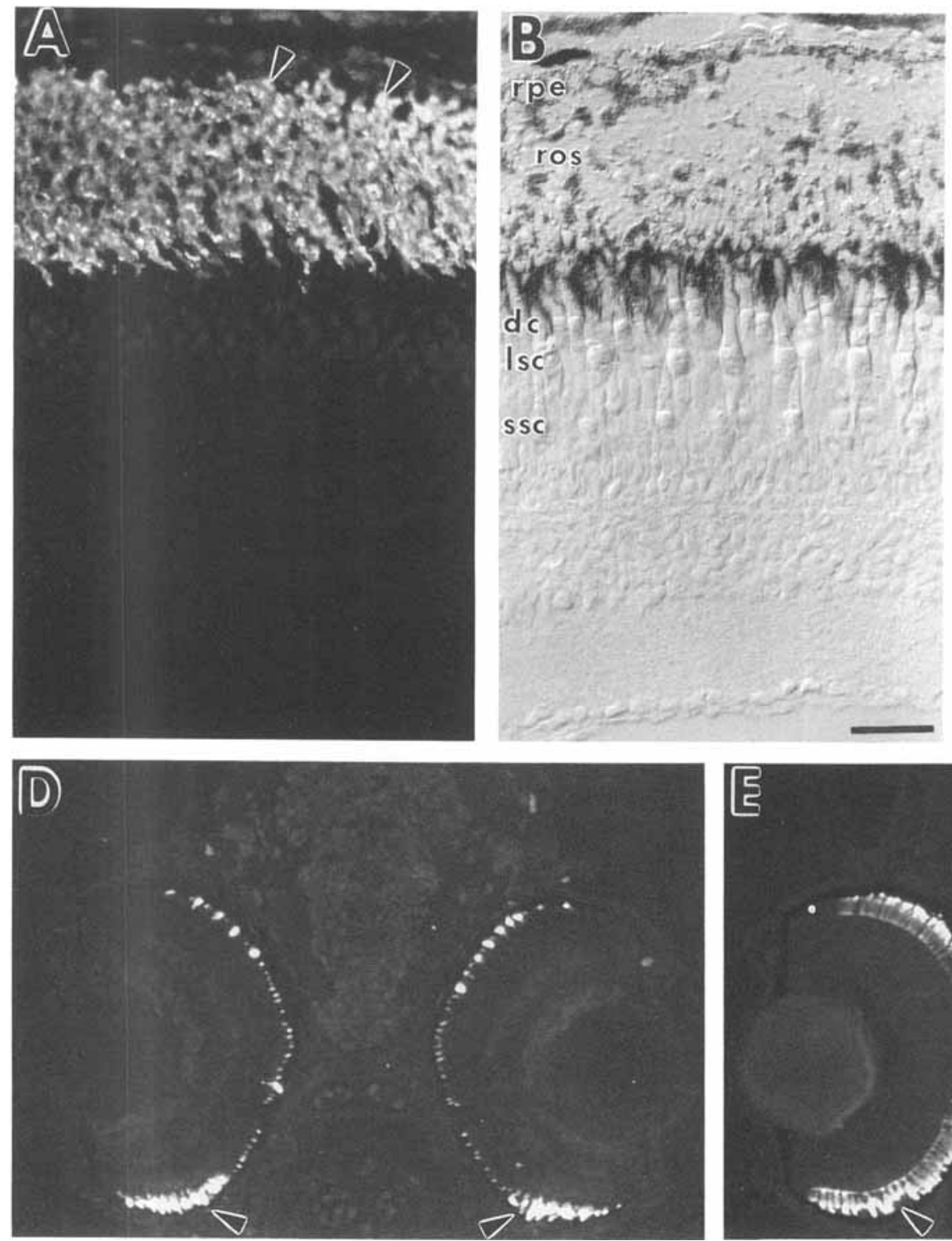

Fig. 2. A: Immunofluorescent section of adult zebrafish retina labeled with ROS1. The fluorescent profiles are rod outer segments (arrowheads). B: Cryosection of adult zebrafish retina viewed with Nomarski optics. ros, rod outer segments; dc, double cones; lsc, long single cones; ssc, short single cones; other abbreviations as in Figure 1. Bar $=25 \mu \mathrm{m}$; also applies to A,C. C: Immunofluorescent section (same section as in B) labeled with FRet43. The double cones are labeled along the entire plasma membrane, and the cone outer segment (cos) is especially bright. D: Immunofluorescent, frontal section through the

sometimes a cluster of rods in ventrotemporal retina, across the choroid fissure from the primary ventral patch.

Stage 4-Density of rods increased in the ventrotemporal patch, scattered rods also at higher density with an obvious secondary cluster of rods in ventrotemporal retina.

Stage 5-ventrotemporal and ventronasal patches at equivalent density and fused across the fissure to form a dumbbell shape.

Stage 6-Ventral patch composed of a continuous swatch of rods at very high density, subtending approxi-

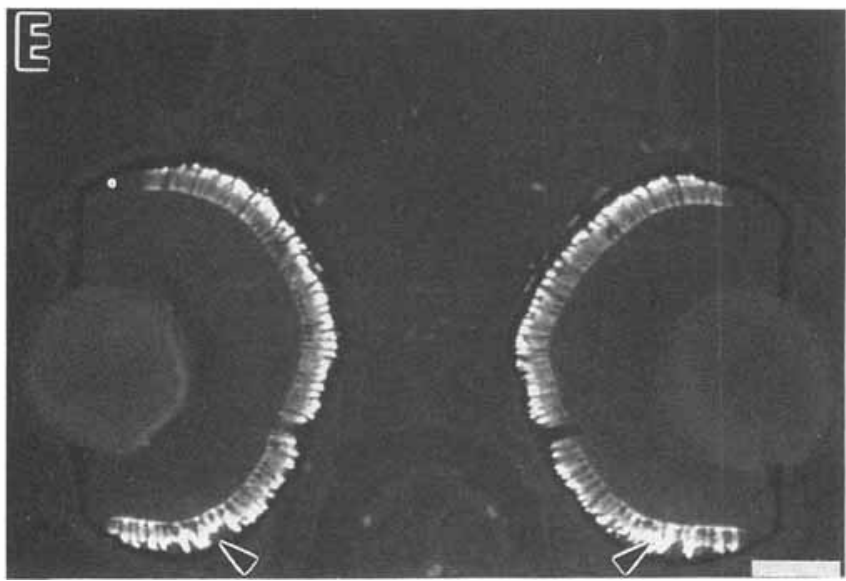

head of a newly hatched zebrafish at 3.5 days PF, stained with ROS1. The stained rod outer segments line the entire retinal hemisphere in each eye. Note that in ventral retina, the rod outer segments are longer and more densely packed (arrowheads). E: Immunofluorescent, frontal section through the head of a newly hatched zebrafish at 3.5 days $\mathrm{PF}$ stained with FRet43. The stained double cones line the entire retinal hemisphere in each eye, at equal density throughout. Note that in ventral retina, the brightly stained outer segments appear slightly longer (arrowhead). Bar $=50 \mu \mathrm{m}$; also applies to D.

mately $150-200 \mu \mathrm{m}$, and the density of rods outside the patch further increased.

In Figure 5 the developmental progression of rods is plotted for 106 eyes examined at 50-84 hours PF and staged according to the above-described series. This histogram, and the corresponding drawings in Figure 4, show that over a period of approximately 1.5 days, differentiation of rod photoreceptors is initiated in ventral retina and then distributed throughout the retinal primordium. Note the variability in stage of rod development for embryos of equivalent age. The variance was most extreme at 56 hours 

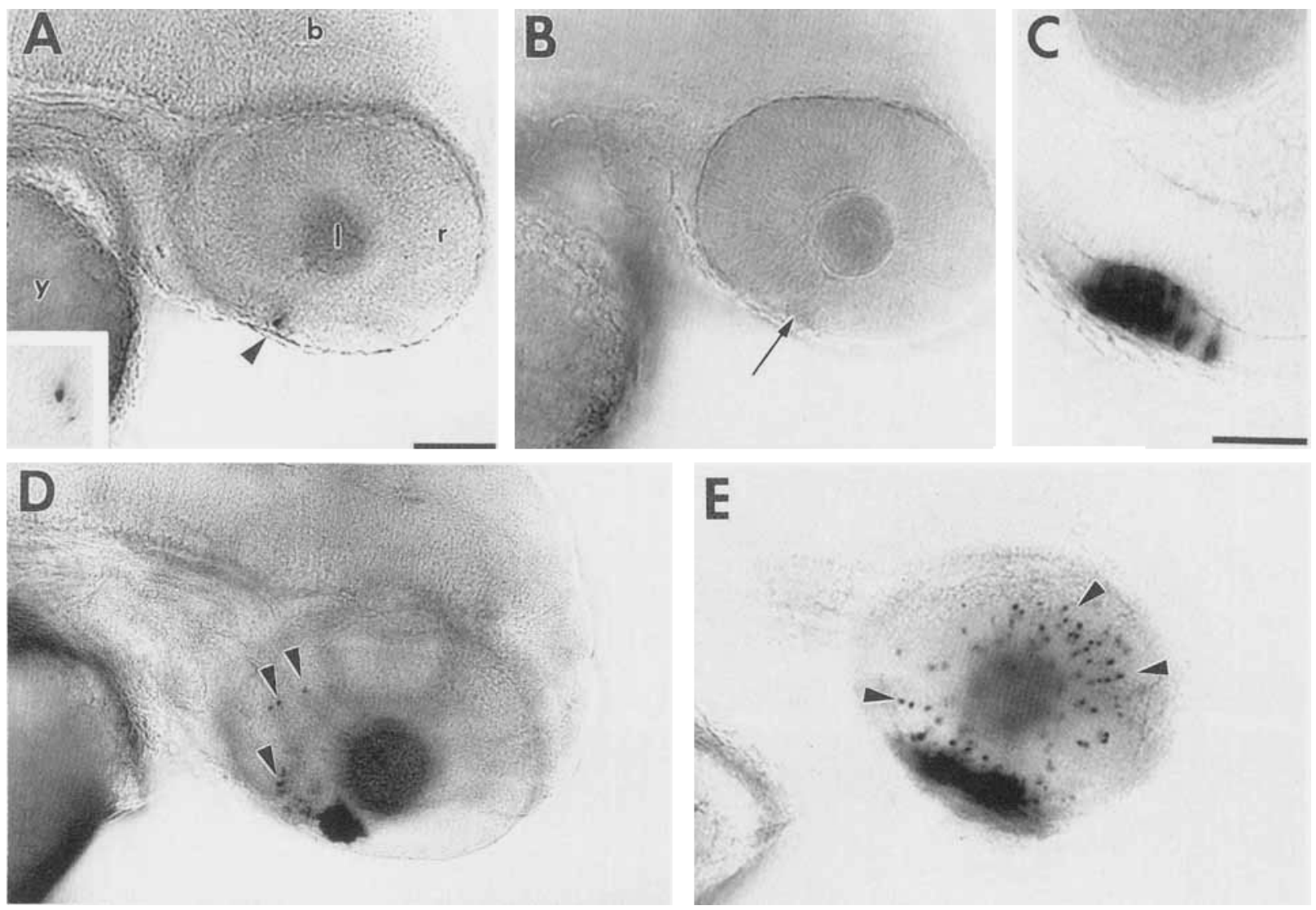

Fig. 3. Whole-mount in situ hybridization with the GFrod probe. A: 50 hour PF. Nasal is to the right. A single rod in ventral retina hybridized with the probe (arrowhead). y, yolk sac; other abbreviations as in Figure 1. Inset: Higher magnification of the labeled rod. Bar $=50$ $\mu \mathrm{m}$; also applies to B, D, and E. B: Same embyro as in A, focused on the

surface of the retinal primordium; note the choroid fissure (arrow). C: ( 6 hour PF; patch of labeled rods. Bar $=25 \mu \mathrm{m}$. D: 52 hour PF; patch of labeled rods with scattered rods in temporal retina (arrowheads). E: 60 hour PF; note radiating rows of rods (arrowheads).

$\mathrm{PF}$, in which developmental stages ranged from 1 to 5 . This variation reflects differences in rate of development among individuals, which is typical of fish embryos, although some of the variance especially for the intermediate and late stages (stages 3-6) may also be due to errors in categorizing embryos on this qualitative, subjective scale.

There appeared to be a marked difference in the pattern of rod differentiation within and outside the region of the early-differentiating ventral patch. Up to 84 hours $P F$, rods in the retina outside the ventral patch never reached a density equivalent to those in the patch. The dorsoventral difference in rod density in embryos can be seen in Figure $6 \mathrm{~A}$, which is a projected series of confocal images of an 84-hour-PF embryo in which the hybridization signal was visualized with Vector Red. The view is from the front of the eye, and note the large discrepancy in rod density between dorsal and ventral hemispheres. Note also that the hybridization signal is less intense for rods that are closer to the central retina; we believe this reflects limited penetration of the probe (also see below). In the eyes in which the NBT/BCI alkaline phosphate substrate was used there was typically less diminution in signal strength within rods in central retina (data not shown). This may reflect increased sensitivity with the NBT/BCI compared to the Vector Red color reaction.

At 84 hours PF, the rods in the ventral patch were more differentiated than those outside the patch, as reflected in the length of the outer segment seen with ROS1 immunostaining (Fig. 2C). Thus the rods within the patch were distinguished from those outside it both by density and level of maturation. Although there was no overall systematic pattern in the distribution of rods outside the ventral patch, there was sometimes a local order, in that three or more rods were aligned in a row which typically radiated from the retinal center (Fig. 3E). This orientation may reflect the underlying organization of proliferative rod precursors that give rise to the new rods, or alternatively, it may be related to the organization of cones (see Discussion).

To summarize, rods outside the ventral patch appeared sporadically and accumulated slowly with an uneven distribution and without an overall pattern, whereas within the ventral patch there was a rapid and localized explosion of rods which quickly (within a few hours) reached very high levels of density and differentiated rapidly.

\section{Cones also originated in the ventral patch and secondarily in nasal retina, but subsequently accumulated evenly throughout the retina}

For the goldfish blue and red cone opsin RNA probes applied to whole-mounted zebrafish embryos, the best results were obtained with tissue fixed in a combination of paraformaldehyde and glutaraldehyde, and digested for 1-2 hours in proteinase K (Uehara et al., 1993). Despite re- 


\section{Stages of Rod Recruitment}
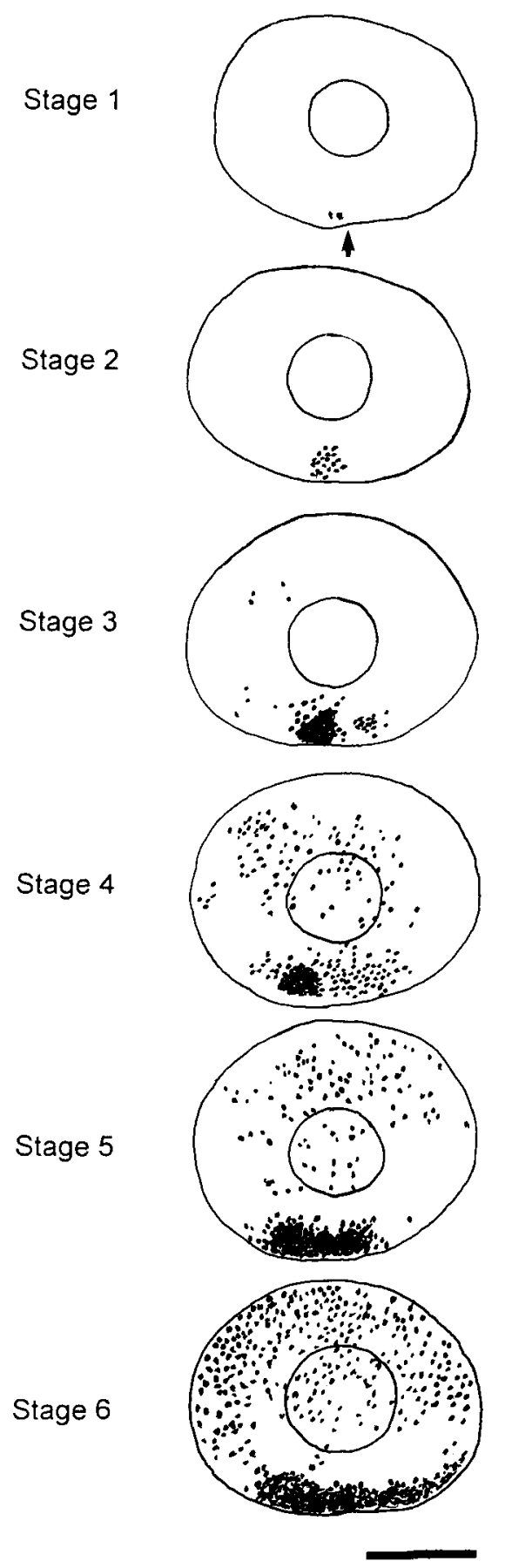

Fig. 4. Stages of rod recruitment. Nasal is to the left. The arrow indicates the location of the choroid fissure. These drawings were made with a camera lucida, by focusing through the eye, and therefore represent a series of projected images. Bar $=100 \mu \mathrm{m}$.

peated attempts to tweak various steps in the hybridization procedure, however, the cone probes were never as reliable as the rod opsin probe, and background staining was typically higher. When the experiments worked, the staining was robust. The results reported for the cone opsins are

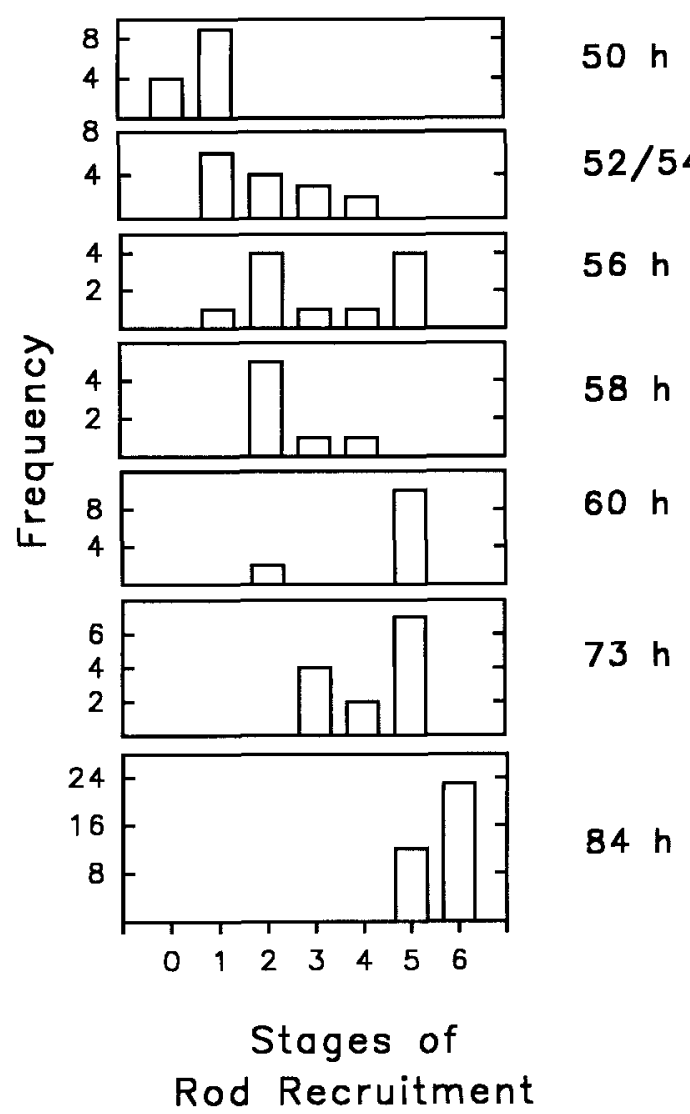

Fig. 5. Histograms showing stage of rod recruitment as a function of embryonic age (hours PF).

based on 9 separate in situ hybridizations (out of a total of 17 performed; in all 17 the rod opsin probe gave positive results). cRNA probes transcribed from either of the available goldfish green cone opsin cDNA clones (GFgr-1 and GFgr-2; Johnson et al., 1993) were unreliable on zebrafish embryos (data not shown), although these same probes hybridized to the appropriate spectral class (the accessory members of the double cone pair) in cryosections or wholemounts of adult zebrafish retina (Raymond et al., 1993a).

The first opsin-expressing blue and red cones appeared in the same retinal location as the first opsin-expressing rods-in a local patch in ventral retina just nasal to the choroid fissure (Fig. 7A,B). The earliest developmental age at which we observed hybridization with the cone opsin probes was 52 hours PF, i.e., 2 hours after the first rods expressed opsin. Again, not all eyes at 52 hours PF contained opsin-expressing photoreceptors, and those that did had only a few. In general there were more labeled cones in older embryos, but we saw the same variation in numbers of cones among embryos of the same age that we had seen for rods. However, the pattern of spread of differentiated cones was very different from that of rods. One difference was that a secondary patch which contained a few labeled cones appeared in nasal retina, separated by about $150 \mu \mathrm{m}$ from the ventral patch (Fig. 7C,D). In addition, once the nasal and ventral patches were established, cones were subsequently added contiguously, that is, immediately adjacent to previously differentiated cones, such that within all regions containing cones the density was approximately constant. 

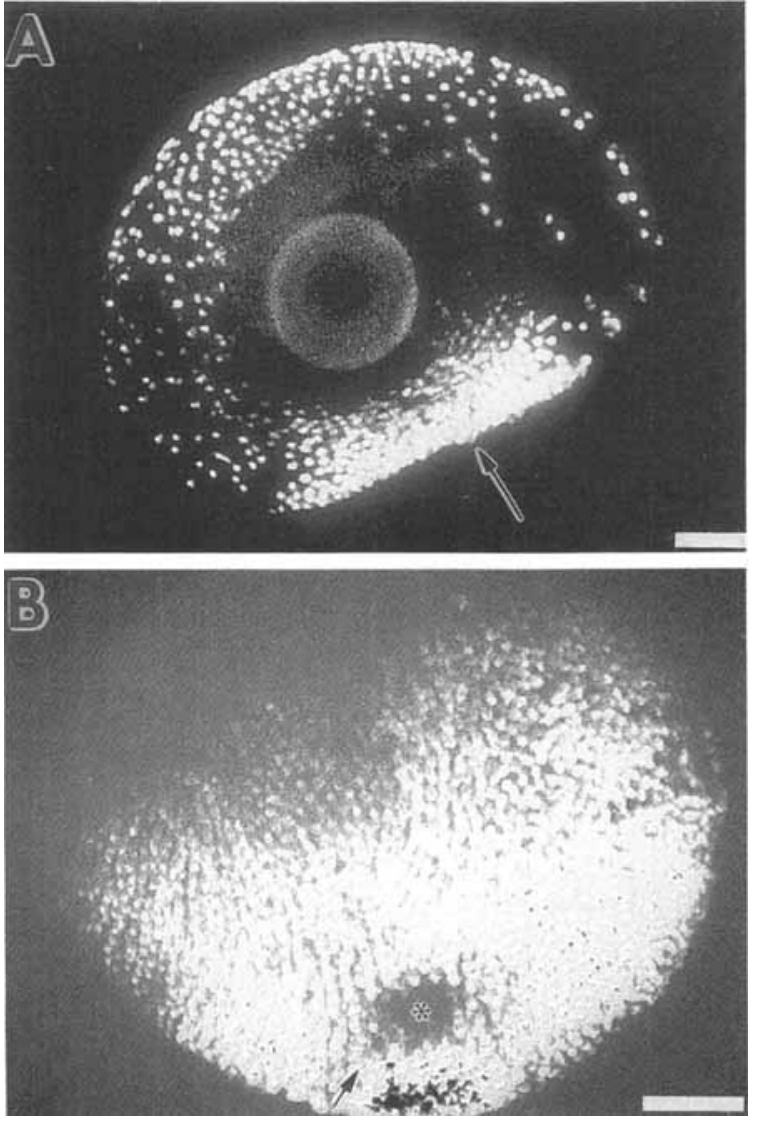

Fig. 6. A: Laser confocal image from Vector Red (fluorescent) in situ hybridization with the GFrod probe on a whole-mount zebrafish embryo at 84 hours PF. Dorsal is up obliquely to the left. This is a series of 14 images, projected in the $z$-axis and viewed from the front of the eye. Bar $=50 \mu \mathrm{m}$. B: Laser confocal image from an immunofluorescent whole-mount of a 73-hour-PF zebrafish eye, stained with FRet43. This is a series of 33 images, projected in the z-axis, and viewed from the back of the eye. This is a black-and-white print of a pseudocolored image; the intensity scale (ascending) is represented by grey to white to black. Nasal is to the right, the arrow indicates the location of the choroid fissure, and the "*" indicates the optic disc. Bar $=50 \mu \mathrm{m}$.

To quantify results with the cone probes, we staged the eyes as we had done for the rod probe. Figure 8 is a series of camera lucida drawings from six representative eyes, ordered from least to most mature. The spatiotemporal pattern of maturation was approximately the same for both red and blue cones and was defined by the stages described below. Again, the criteria used were chosen primarily for ease of description, and although they are similar to those used to categorize stages of rod development, in a given area of retina there were fewer cones of a specific subtype compared to rods. Thus the precocial patch of cones (stage 1) that was roughly equivalent in size to the patch of rods had fewer labeled cells.

Stage 0-No cells express cone opsin.

Stage 1-Fewer than 10 labeled cones in a ventral patch.

Stage 2-Two patches of less than 10 labeled cones each, one ventral and the other in nasal retina.

Stage 3-The ventral and nasal patches fused, filling the ventronasal sector.
Stage 4-One half of the retina contained labeled cones, centered on the ventronasal pole.

Stage 5-Three-fourths of the retina contained labeled cones, with only the dorsotemporal sector empty.

Stage 6-The entire retina contained labeled cones.

For the blue opsin probe, 96 eyes at 52-84 hours PF were staged according to the above categories; the results are plotted in Figure 9. At 52 hours PF, only one of nine eyes had a hybridization signal, and there appeared to be a single heavily labeled cone accompanied by a few less heavily labeled ones (Fig. 7B); the other eight eyes were negative (e.g., Fig. 7A). In the eye which contained the labeled blue cone, the outer nuclear layer was recognizable as a single row of columnar cells, which extended over a substantial fraction (about three-fourths) of the circumference of the retinal margin (Fig. 7B). In another 52-hour-PF eye, processed for in situ hybridization in the same tube, there was no hybridization signal (Fig. 7A). In this eye the outer nuclear layer had just begun to laminate, and a row of only a few presumptive photoreceptors could be seen in a small patch in ventral retina. These discrepancies in the state of histological maturation among eyes support the inference that the variability in opsin signals was due to individual differences in developmental rates.

With increased time postfertilization, increased numbers of embryos were at more mature stages of blue cone development (Fig. 9), similar to what was seen for rods (Fig. 5). Again, there was a large variance, especially at 73 hours $\mathrm{PF}$, in which all stages were represented. Moreover, at almost all ages PF some embryos failed to show an opsin signal (stage 0). We have no explanation for this. By 84 hours PF, most of the embryos were at stage 5 or 6 .

For the red cone opsin probe, we had fewer time points (and a total of 89 eyes), but the overall pattern of maturation was the same as for the blue cone opsin probe (Fig. 10). Figure 7C,D shows ventral views of a 53-hour-PF embryo at stage 2, with the focus on the ventral patches $(C)$ or the nasal patches (D). By 84 hours PF, most eyes (32 out of 39) were at stage 6 , and red cone opsin expression had spread all the way to the margin of the retinal primordium and along the entire retinal circumference. Figure $7 \mathrm{E}, \mathrm{F}$ shows lateral views of an 84-hour-PF eye, focused first on the ventronasal side and then on the dorsotemporal side, respectively. Although there were only a few labeled red cones at the dorsotemporal pole, it was clear that a ring of opsin-expressing red cones surrounded the eye (Fig 7F). The penetration problem seen at 84 hours $\mathrm{PF}$ with the rod opsin probes was even more obvious here. Whereas at earlier ages opsin-expressing cones were found in central retina, at 84 hours $\mathrm{PF}$ the signal faded out rapidly from periphery to center, and at the back of the eye there were no labeled cones (Fig. 7E,F). We conclude that at 84 hours PF the eye developed a barrier to penetration of the probes, and because the whole-mounts were processed with the eyes in situ, it is likely that the barrier arose with maturation of the epidermis.

From the time when they first appeared, the blue and red cones were distributed with a regular spacing indicative of a mosaic pattern. Unlike the sporadic and irregular appearance of rods outside the ventral patch, both blue and red cones accumulated by a progressive enlargement of the differentiated region, and within this region, cone spacing was roughly constant. The distance between adjacent cones along rows orthogonal to the retinal margin (measured on 


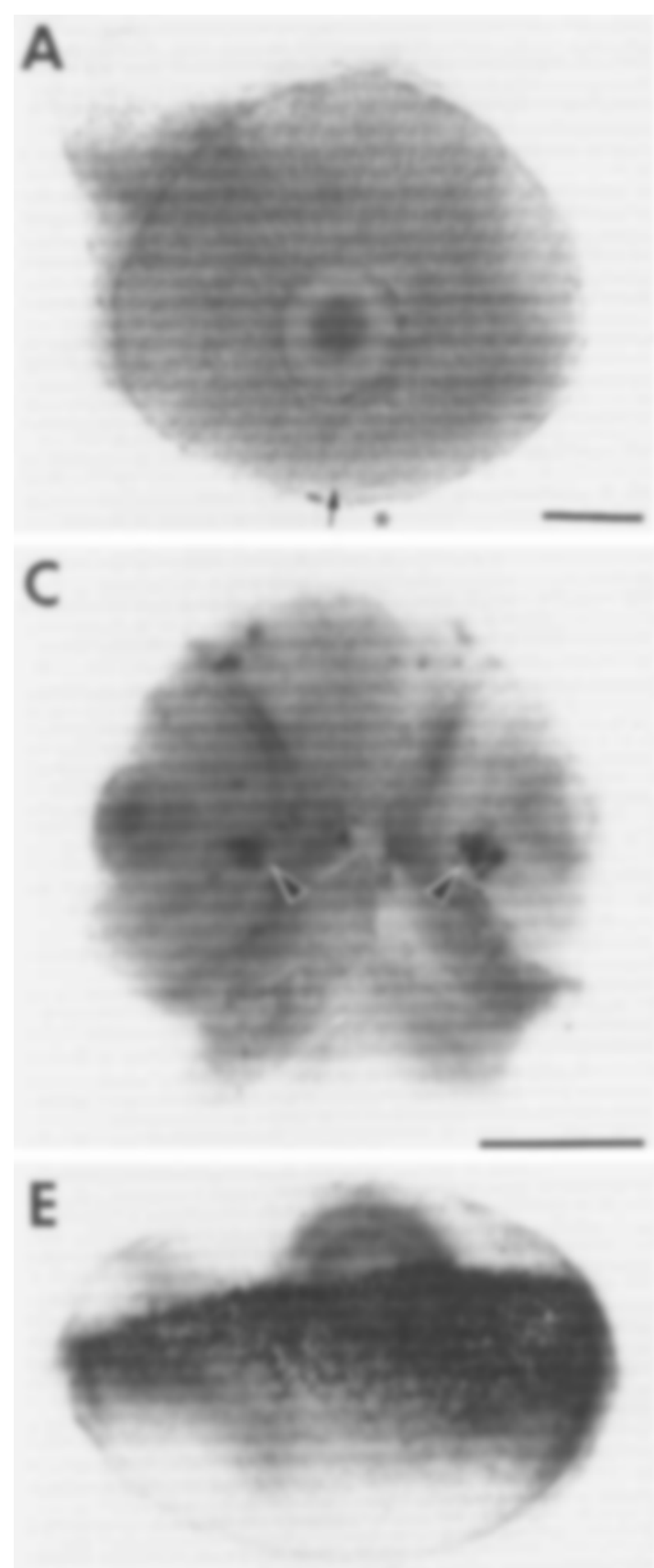

Fig. 7. Whole-mount in situ hybridization with cone opsin probes. A: 52 hours PF, GFblu probe. Nasal is to the right. On the nasal side of the choroid fissure (arrow), the outer nuclear layer has begun to form $\left.{ }^{*}\right)$, but there is no hybridization signal. Bar $=50 \mu \mathrm{m}$; also applies to $\mathrm{B}$, E, F. B: 52 hours PF, GFblu probe. The outer nuclear layer extends around three-fourths of the circumference of the eye (indicated by *); and one blue cone (arrowhead), in ventral retina, is heavily labeled and a few nearby ones are lightly labeled. C,D: 53 hours PF, GFred probe.

camera lucida drawings at 84 hours PF) was $5.44 \pm 0.93$ $\mu \mathrm{m}$ and $6.05 \pm 1.17 \mu \mathrm{m}$ for blue cones $(\mathrm{n}=40$ and $\mathrm{n}=49$, respectively, measured on two eyes) and $4.75 \pm 0.81 \mu \mathrm{m}$ and $4.74 \pm 0.38 \mu \mathrm{m}$ for red cones $(n=18$ and $n=10$, respectively, measured on two eyes). Blue cones were farther apart, since on average, the distance between neighboring
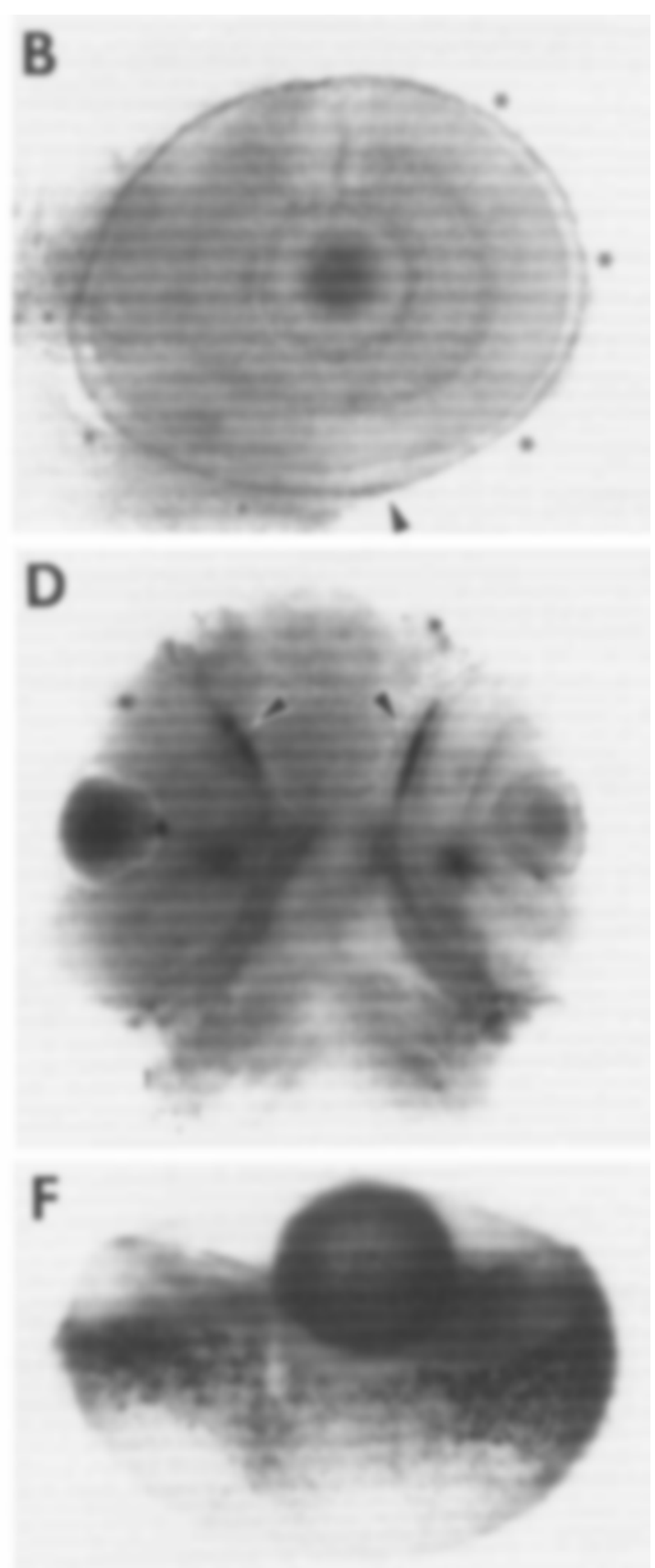

Whole head, viewed from the ventral side, nasal is up. In $\mathrm{C}$ the focus is on patches of labeled red cones (arrowheads) in ventral retina. The labeled cones are viewed "end on,"and appear as dots. Bar $=100 \mu \mathrm{m}$; also applies to $D$. In $D$ the focus is on the labeled patch in nasal retina (arrowheads), and the cones are viewed longitudinally. E,F: 84 hours $\mathrm{PF}, \mathrm{GFred}$ probe. This isolated eye is viewed from the side. In $\mathrm{E}$ the focus is on the ventronasal side; in F, on the dorsotemporal side.

cones was $5.78 \mu \mathrm{m}$ for blue versus $4.75 \mu \mathrm{m}$ for red, and the ratio (intercone spacing for blue cones $\div$ intercone spacing for red cones) was 1.22. For comparison, on a wholemounted adult zebrafish retina prepared for in situ hybridization with the blue opsin probe (data not shown), blue cones were spaced at $9.19 \pm 1.15 \mu \mathrm{m}(\mathrm{n}=43)$, and on 


\section{Stages of Cone Recruitment}

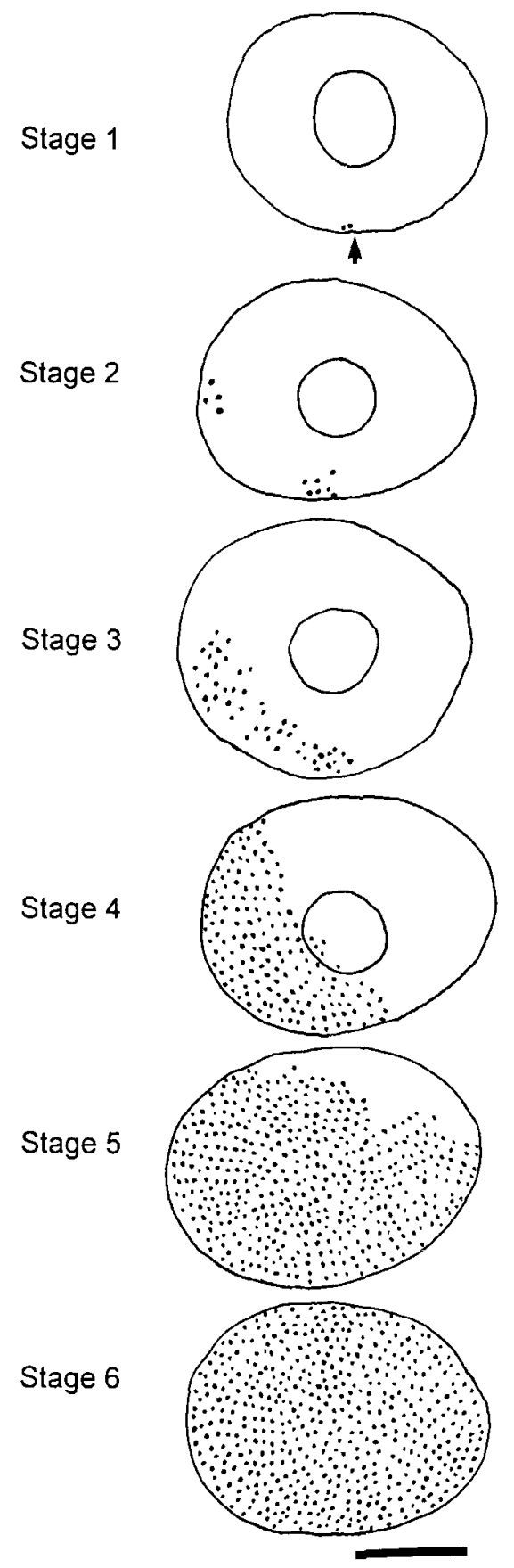

Fig. 8. Stages of cone recruitment. Nasal is to the left. The arrow indicates the location of the choroid fissure. Stages 1-3 are camera lucida drawings of embryos hybridized with the red opsin probe and viewed from the front of the eye. Stages 4-6 are composite drawings from camera lucida and photomontages of preparations hybridized with either the red or the blue opsin probe and viewed from the back of the eye. Bar $=100 \mu \mathrm{m}$.

another retina hybridized with the red opsin probe, red cones were separated by $5.25 \pm 0.81 \mu \mathrm{m}(\mathrm{n}=64)$, and the ratio of intercone spacing (blue:red) in the adult was 1.75 .

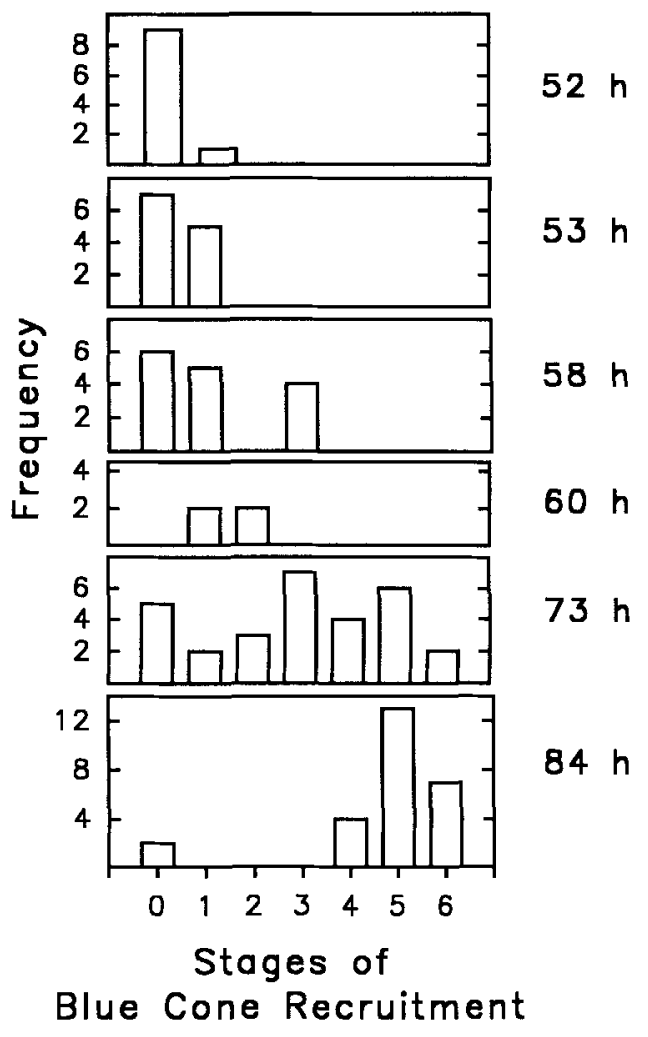

Fig. 9. Histograms showing stage of blue cone recruitment as a function of embryonic age (hours PF).

Additional information about the progression of cone differentiation was obtained from FRet43-immunofluorescent whole-mounts. Figure $6 \mathrm{~B}$ shows a projected series of confocal images from a 73-hour-PF embryo. Because FRet43 labels the entire plasma membrane, the most mature cones (the ones which had developed an outer segment, i.e., those in the ventral patch) were the most heavily labeled, and the secondary patch which was seen in nasal retina with the opsin probes was also apparent in these preparations as a region of slightly more mature cones (Fig. $6 \mathrm{~B}$ ). The overall ventronasal-to-dorsotemporal gradient of cone maturation is obvious. For the most part, the FRet43-labeled cones were aligned in rows, and the cones outside the ventral patch appeared evenly distributed. The even distribution of cones was also easily appreciated in FRet43-immunostained retinal sections (Fig. 2E).

This impression that FRet43-labeled cones were equally spaced was verified by measuring the intercone spacing along rows orthogonal to the retinal margin; these measurements were made on photographs of the confocal projection series shown in Figure 6B. The average distance between neighboring cones in each of the four retinal quadrants (dorsotemporal, dorsonasal, ventrotemporal, and ventronasal) is plotted in Figure 11. These data show that the Fret43-labeled cones are spaced equally throughout the retina. The density of cones within the patch could not be measured because the intense fluorescent signal in the outer segments (see Fig. 2C,E) obscured the separation between individual cells. Outside the patch, the FRet43labeled profiles appeared circular and discrete, and it was not clear from these images whether each profile represented a double cone pair (i.e., red and green members), or 


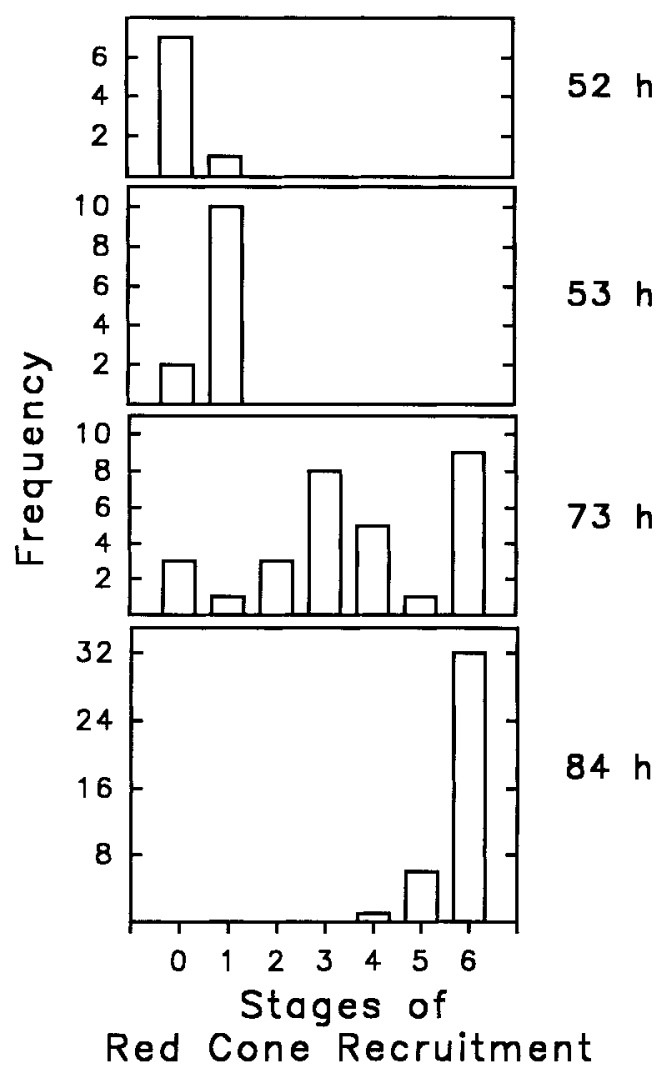

Fig. 10. Histograms showing stage of red cone recruitment as a function of embryonic age (hours PF).

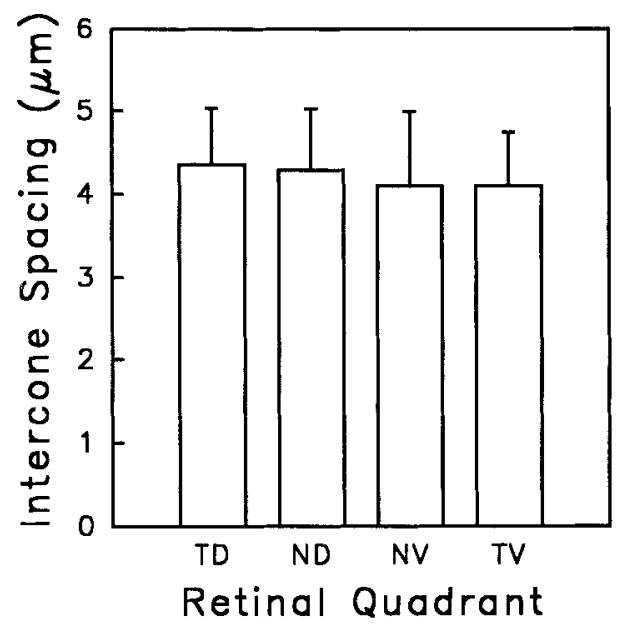

Fig. 11. Spacing of FRet43-labeled cones in a 73-hour-PF embryo. See text for details.

whether the double cone members had not yet fused, and each profile represented an individual cone (see Discussion).

In summary, expression of cone opsins was initiated at 52 hours PF in the ventral patch where rods originated, and secondarily in a small region in nasal retina. The accretion of additional cones by a crystallization-like process then led to fusion of the two patches, and the zone of differentiated cones subsequently enlarged in the dorsotemporal direction until it reached the retinal margin. The entire retina was filled with cones by about 84 hours PF, and from the beginning, both red and blue cones were arrayed in a mosaic pattern, equally spaced throughout the retina.

\section{DISCUSSION}

That the first photoreceptors to express opsin in the zebrafish retina are located in ventronasal retina is not surprising, as it had already been shown that in embryonic zebrafish retina, neuronal differentiation, including photoreceptors (Kljavin, 1987; Larison and BreMiller, 1990) and ganglion cells (Burrill, 1993) begins in ventral retina adjacent and nasal to the choroid fissure. By the use of specific opsin probes we have extended this work to include a comparison of the spatiotemporal patterns of maturation of rods, blue cones, and red cones. We found that all three types of photoreceptors originated in this ventral patch, although subsequent spatiotemporal patterns of differentiation differed between rods and cones, whereas blue and red cones appeared to follow the same pattern.

The significance of the precocial differentiation of retinal cells at the ventral margin of the retina in embryonic zebrafish, and the mechanisms responsible for it, are uncertain. The discrete nature of the precocial patch is consistent with an inductive process (Saha et al., 1992), and its location adjacent to the choroid fissure suggests a possible relationship with major morphogenetic events that occur during early eye development (Schmitt and Dowling, 1994). At the earliest stages of ocular morphogenesis in zebrafish, the choroid fissure (and optic stalk) are located at the anterior border of the eye, but between 24 and 36 hours $\mathrm{PF}$ the eye rotates so that the choroid fissure becomes positioned at the ventral pole (Schmitt and Dowling, 1994). During this period, the first ganglion cells begin to differentiate (Burrill, 1993), and about 14 hours later, the first rod photoreceptors differentiate in the same region (present data). Dowling and colleagues (Hyatt et al., 1992) have also recently demonstrated that exposure of zebrafish embryos to retinoic acid at 9-11 hours PF produces a duplicated retina which appears to arise from this same region adjacent to the optic stalk/choroid fissure (Schmitt and Dowling, 1994). Because at the time of induction this region was anterior in the head, this result is consistent with the known role of retinoic acid in anterior-posterior patterning and with the retinoic-acid sensitivity of an anterior domain in the early embryo (Saha et al., 1992). Whether the early-differentiating patch of ventral retina is related in any way to the retinoic-acid-sensitive domain is unknown, but it would be interesting to examine the pattern of photoreceptor differentiation in duplicated retinas of embryos exposed to retinoic acid. It is also interesting to note that the position of the optic stalk at the ventral margin of the retina in the zebrafish embryo is a transient, developmental phenomenon, since in the adult, the optic disc is centrally positioned. Although the later stages of postembryonic retinal growth and cell proliferation have not been investigated in zebrafish, studies in other teleosts, such as goldfish, have shown that the relative position of the optic disc shifts toward central retina as a result of asymmetric growth due to an increased level of proliferation in ventral compared to dorsal retina (Raymond, 1986). Therefore, it is likely that the earliest born photoreceptors in the zebrafish retina eventually reside in the central retina, consistent with the central-peripheral gradient of maturation characteristic of vertebrate retinal development (Stone, 1988). 
Early stages of rod opsin expression have also been examined in Xenopus embryos with a Xenopus rod opsin cDNA probe (Saha and Grainger, 1993) and in newt embryos with a rod opsin antibody (McDevitt et al., 1993). In both studies, opsin expression first appeared in a few cells located in the central retina, and with increased development, the area containing opsin-expressing rods expanded toward the margins, although details concerning maturation of the pattern of rod opsin expression were not provided. In the present study, we found that although rod opsin expression eventually spread across the embryonic zebrafish retina, at all developmental stages, rod density in ventral retina was higher than in dorsal retina, just as it is in adult zebrafish (Nawrocki, 1985; P.A. Raymond and S.-Y. Lin, unpublished observations). To our knowledge, this is the first demonstration that differential cell proliferation might account for topographic differences in numbers of retinal cells. In contrast, mechanisms based on cell death or cell migration or differential retinal stretching, rather than cell proliferation, have been shown to shape cell density profiles in mammalian retinas (Lia et al., 1987; Robinson, 1987; Wong and Hughes, 1987; Packer et al., 1990).

Retinal cell birthdating studies with ${ }^{3} \mathrm{H}$-thymidine, carried out in many species, including mammals (Stone, 1988) and teleost fish (Raymond, 1991; Fernald, 1991), have shown that cones are generated (i.e., undergo their terminal mitotic division) before rods. Therefore it was surprising that in the present study rod opsin appeared first, at 50 hours PF, whereas expression of both blue and red cone opsins began 2 hours later. We have not done birthdating studies in combination with in situ hybridization of opsin probes, so we cannot say whether the earliest differentiating rods in the ventral patch were generated before or after the cones in the same location. The earliest rods seemed unusual in that their pattern of accumulation differed from that in more distant regions of retina. Outside the ventronasal patch, rods accumulated sporadically and slowly, consistent with the established mechanism of delayed rod genesis in larval and adult teleost fish retinas, in which production of rod photoreceptors occurs subsequent to the generation of cones through continued proliferation of neuroepithelial cells called rod precursors (Raymond, 1985a, 1991; Fernald, 1991). From the present data it is not clear whether the rods in the ventronasal patch arose from rod precursors as traditionally defined or through a different, as yet unidentified, mechanism.

Another unusual feature of the earliest rods was their regular arrangement, which contrasted with the lack of an obvious pattern of rods in the mature retina. A mundane explanation for this observation is that the organization of these first rods may have been imposed by the orderly spacing of cones, assuming that the cone mosaic pattern had already been established (Lyall, 1957; Kunz et al., 1983; Larison and BreMiller, 1990). A more exciting alternative is that the precocial rods may play a primary role in organizing the cone mosaic pattern, a suggestion which is supported by data from similar studies in developing goldfish retina (Stenkamp and Raymond, 1994). Data presented here, in the histograms of developmental stage of opsin expression as a function of embryonic age (Figs. 5, 9, 10) are also consistent with this hypothesis. The histograms show not only that rod opsin is the first to be expressed, but that it spreads more quickly across the retina. Note that within a few hours after rod opsin expression began (stage 1) rods expressing opsin could be found throughout the retina (stage 5) in at least some of the eyes (Fig. 5). In contrast, spread of blue and red cone opsin expression initially began slowly, then progressed more rapidly (Figs. 9 and 10), as a wave of differentiating cones eventually overtook the rods.

These results, together with the earlier birthdating studies, would imply that cones differentiate more slowly than rods, at least with respect to expression of opsin message. This conclusion is consistent with immunocytochemical studies in fetal monkey retina, which showed that expression of rod opsin protein preceded that of the cone opsins, even though cones in the monkey retina are born before rods (Bumsted et al., 1994). Also consistent with this conclusion is evidence that maturation of cones is dependent on rods, since cones failed fully to differentiate in transgenic mice in which rods were ablated with a cytotoxic gene driven by a rod-specific promoter (Usukura et al., 1993, 1994). On the surface, our present findings seem inconsistent with previous studies of photoreceptor cytodifferentiation in goldfish (Raymond, 1985b) and zebrafish (Branchek and BreMiller, 1984), which found that morphological maturation of cones is more rapid than rods; in contrast, in guppies, formation of outer segment discs was more rapid in rods than in cones (Kunz et al., 1983). However, since photoreceptors begin to express opsin before cytodifferentiation is complete, and even before outer segment formation begins (Araki, 1984; Hicks and Barnstable, 1987; Knight and Raymond, 1990; Matesic et al., 1994), we might not expect molecular and morphological indices of photoreceptor maturation to be completely synchronized.

Although we had expected that these opsin probes would provide us with equivalent reagents to examine rod and cone development, some caution is warranted in the above interpretation of these results, since technical limitations of the in situ hybridization experiments were more obvious with the cone probes than with the rod probe. For example, we cannot exclude the possibility that the apparent delay of 2 hours in the appearance of cone opsin expression and the apparently slower rate of spread across the retina may have been due to decreased sensitivity of the cone probes, or alternatively, to higher levels of opsin expression in young rods compared to cones. The differences between the rod and cone opsin probes are not likely to be due to differences in the degree of homology between the goldfish and zebrafish rod and cone opsins, since the overall levels of nucleotide homology are similar. Additional studies with sectioned material (in which probe penetration would not be as limiting) and with additional photoreceptor-specific probes will be necessary to resolve this uncertainty completely.

The most important fundamental observation of this study came from measurements of intercone spacing, which suggested that the cones are arranged in a mosaic pattern from the time of initiation of opsin expression, with red cones spaced more closely than blue cones, as they are in the adult zebrafish cone mosaic. This implies that cell identity and cell position are related, consistent with a potential mechanism whereby lateral, inductive signals might determine photoreceptor identity in teleost fish, similar to what has been shown in Drosophila (Cagan and Zipursky, 1992). Some additional refinement of the teleost cone mosaic may take place at later developmental stages, since in embryonic zebrafish the blue cones were more closely spaced relative to red cones than they were in adults. In a recently published correspondence, Szél and colleagues (Szél et al., 1994) argue that in developing rat retina, green cones arise from cones which initially express blue opsin. However, the highly regular mosaic patterns of zebrafish cones we ob- 
served from the earliest stages of differentiation argue against the switching of opsin phenotype as a mechanism for refinement of the zebrafish cone mosaic. In other teleosts, the cone mosaic undergoes more substantial alterations in organization during later stages of retinal growth as a result of shifting and rotation of the individual elements (reviewed in Raymond, 1995). Although nothing is known about the precise mechanisms involved, these rearrangements only alter relative spacing and do not necessarily involve the breaking and reestablishment of neighbor relationships. Therefore it is likely that these refinements result from positional displacements in the plane of the retina rather than phenotypic alterations in spectral subtype. Interestingly, such lateral movements of photoreceptors have been shown to occur in the final stages of development in primate retina, during formation of the fovea (Packer et al., 1990). Another important event in the differentiation of teleost cones also involves a change in relative spacing: the fusion of red and green cones to form the double cone pairs. Branchek and BreMiller (1984) reported that double cones were not identifiable until 12 days PF in zebrafish; however, Larison and BreMiller (1990), using their FRet43 monoclonal antibody, reported that double cones were present as fused pairs by 48 hours PF. Our measurements of intercone spacing of FRet43labeled cones (on average, $4.37 \mu \mathrm{m}$ ) compare favorably with the spacing of cones expressing red opsin (on average, 4.75 $\mu \mathrm{m}$ ), which is consistent with the conclusion that the red/green pairs are fused at this age.

We do not yet have data for the remaining two spectral cone subtypes, green and ultraviolet, although another recent study (Robinson et al., 1994) has shown that the pattern of differentiation of ultraviolet cones in zebrafish is similar to that of blue and red cones. These authors also suggest that ultraviolet cones are the first to express opsin, although their putative zebrafish ultraviolet pigment is unusual in that by sequence homology it is more closely related to vertebrate rhodopsins than to the cone pigments (Robinson et al., 1993). The data presented here suggest that rod opsin is expressed first, and there is some hint that expression of red opsin might precede blue, since at all comparable ages, a higher proportion of eyes were categorized at more mature stages of cone development when hybridized with the red opsin probe compared to the blue. Unfortunately, the variability in overall developmental rate and the extreme rapidity with which the eyes moved from stage 0 to stage 6 of photoreceptor recruitment preclude any firm conclusions about the order of differentiation of cone types in the zebrafish retina from the data presented here. This is a very interesting question, since it has implications for putative inductive mechanisms between cone subtypes that might regulate cell determination and mosaic patterning (Raymond, 1995). A similar study in embryonic goldfish with opsin probes identical to those used here, as well as additional probes for green opsin and a putative goldfish ultraviolet pigment (Hisatomi et al., 1994), showed clearly that in embryonic goldfish rod opsin is expressed first, followed by red, green, blue, and then ultraviolet (Stenkamp and Raymond, 1994). Thus, our data in goldfish and zebrafish are consistent in the apparent order of opsin expression: first rod, then red, then blue. In mammals, this same question is being investigated with $\mathrm{red} /$ green versus blue opsin-specific antibodies, but the results are thus far inconclusive. One group finds that the first cones to differentiate in fetal macaque monkey retina are immunoreactive for red/green opsin, although they have the spacing characteristic of blue cones (Wikler and Rakic, 199, 1994), whereas others working with developing primate (Bumsted et al., 1994) or mouse (Szél et al., 1993) retinas find that blue cones are first. Interestingly, only the blue cones form a regular array in the mammalian retina, whereas red/green cones are less regularly distributed (Marc and Sperling, 1977; de Montasterio, et al., 1981; Szél et al., 1988). In summary, although the order of differentiation of the various spectral subtypes of cones in the vertebrate retina remains uncertain, it is clear that in both fish and mammals, the organization of the cone mosaic pattern is apparent from the earliest stages of photoreceptor differentiation.

\section{ACKNOWLEDGMENTS}

This work was supported by NSF IBN-9222046 to P.A.R. MaryEllen Rounsifer provided valuable technical assistance. University of Michigan undergraduate students SuiYen Lin and Bernadette Angeles participated in some of the preliminary experiments; B.A. was supported by NSF training grant Dir-9014275. G.A.C. was supported by the University of Michigan Medical Scientist Training Program, NIH training grant T32 GM07863. We thank K. Nakanishi for the goldfish opsin clones and R. BreMiller for the FRet43 antibody. We also thank Judith Robinson, Ellen Schmitt, and John Dowling for stimulating discussions.

\section{LITERATURE CITED}

Adler, R. (1993) Determination of cellular types in the retina. Invest. Ophthalmol. Vis. Sci. 34:1677-1682.

Ahlbert, J.B. (1973) Ontogeny of double cones in the retina of perch fry (Perca fluviatilis. L., Teleostei). Acta Zool. 54:241-254.

Ahnelt, P.K., H. Kolb, and R. Pflug (1987) Identification of a subtype of cone photoreceptor, likely to be blue sensitive, in the human retina. J. Comp. Neurol. 255:18-34

Altshuler, D.M., D.L. Turner, and C.L. Cepko (1991) Specification of cell type in the vertebrate retina. In D.M.K. Lam and C.J. Shatz (eds): Development of the Visual System. Cambridge, MA: MIT Press, pp. 37--58.

Araki, M. (1984) Immunocytochemical study on photoreceptor cell differentiation in the cultured retina of the chick. Dev. Biol. 103:313-318.

Barthel, L.K., and P.A. Raymond (1990) Improved method for obtaining 3-micron cryosections for immunocytochemistry. J. Histochem. Cytochem. 38:1383-1388

Barthel, L.K., and P.A. Raymond (1993) Subcellular localization of alphatubulin and opsin mRNA in the goldfish retina using digoxigenin-labeled cRNA probes detected by alkaline phosphatase and HRP histochemistry. J. Neurosci. Methods 50:145-152.

Bennett, H.S., A.D. Wyrick, S.W. Lee, and J.H. McNeil (1976) Science and art in preparing tissues embedded in plastic for light microscopy, with special reference to glycol methacrylate, glass knives and simple stains. Stain Technol. 51:71-97.

Braisted, J.E., and P.A. Raymond (1993) Continued search for the cellular signals that regulate regeneration of dopaminergic neurons in goldfish retina. Dev. Brain Res. 76:221-232.

Branchek, T., and R. BreMiller (1984) The development of photoreceptors in the zebrafish, Brachydanio rerio. I. Structure. J. Comp. Neurol. 224:107115 .

Bumsted, K., C. Lerea, A. Szél, E. Dorn, P. Röhlich, and A. Hendrickson (1994) Appearance of cone and rod opsins in the developing primate retina. Invest. Ophthalmol. Vis. Sci. Suppl. 35:1728.

Burrill, J.D. (1993) The early development of retinal ganglion cell projections in the zebrafish, Brachydanio rerio. Ph.D. dissertation. University of Michigan.

Cagan, R.L., and S.L. Zipursky (1992) Cell choice and patterning in the Drosophila retina. In M. Shankland and E.R. Macagno (eds): Determinants of Neuronal Identity. San Diego: Academic Press, pp. 189-224.

Coutinho, L.L., J. Morris, and R. Ivarie (1992) Whole mount in situ detection of low abundance transcripts of the myogenic factor $\mathrm{qmf} 1$ and myosin heavy chain protein in quail embryos. Biotechniques 13: 722-724. 
de Monasterio, F.M., S.J. Schein, and E.P. McCrane (1981) Staining of blue-sensitive cones of the Macaque retina by a fluorescent dye. Science 213:1278-1281.

Engström, K. (1960) Cone types and cone arrangement in the retina of some cyprinids. Acta Zool. 41:277-295.

Engström, K. (1963) Cone types and cone arrangements in teleost retinae. Acta Zool. 44:179-243.

Fernald, R.D. (1991) Teleost vision: seeing while growing. J. Exp. Zool. Suppl. 5:167-180.

Harris, W A., and S.L. Messersmith (1992) Two cellular inductions involved in photoreceptor determination in the Xenopus retina. Neuron 9:357-372.

Hemmati-Brivanlou, A., D. Frank, M.E. Bolce, B.D. Brown, H.L. Sive, and R.M. Harland (1990) Localization of specific mRNAs in Xenopus embryos by whole-mount in situ hybridization. Development 110:325-330.

Hicks, D., and C.J. Barnstable (1987) Different rhodopsin monoclonal antibodies reveal different binding patterns on developing and adult rat retina. J. Histochem. Cytochem. 35:1317-1328.

Hisatomi, O., S. Kayada, Y. Aoki, T. Iwasa, and F. Tokunaga (1994) Phylogenetic relationships among vertebrate visual pigments. Vision Res. 23:3097-3102.

Hyatt, G.A., E.A. Schmitt, N R. Marsh-Armstrong, and J.E. Dowling (1992) Retinoic acid-induced duplication of the zebrafish retina. Proc. Natl. Acad. Sci. U.S.A. 89:8293-8297.

Johnson, R.L., K.B. Grant, T.C. Zankel, M.F. Boehm, S.L. Merbs, J. Nathans and K. Nakanishi (1993) Cloning and expression of goldfish opsin sequences. Biochem. 32:208-214.

Kljavin, I.J. (1987) Early development of photoreceptors in the ventral retina of the zebrafish embryo. J. Comp. Neurol. 260:461-471.

Knight, J.K., and P.A. Raymond (1990) Time course of opsin expression in developing rod photoreceptors. Development 110:1115-1120.

Kunz, Y W., S. Ennis, and C. Wise (1983) Ontogeny of the photoreceptors in the embryonic retina of the viviparous guppy, Poecilia reticulatus $P$. (Teleostei). An electron-microscopical study. Cell Tissue Res. 230:469486.

Larison, K.D., and R. BreMiller (1990) Early onset of phenotype and cell patterning in the embryonic zebrafish retina. Development 109:567-576.

Lia, B., R.W. Williams, and L.M. Chalupa (1987) Formation of retinal ganglion cell topography during prenatal development. Science 236:848850.

Lyall, A.H. (1957) The growth of the trout retina. Quart. J. Micros. Sci. 98:101-110.

Marc, R.E. (1986) The development of retinal networks. In R. Adler and D. Farber (eds): The Retina: A Model for Cell Biology Studies. New York: Academic Press, pp. 17-65.

Marc, R.E., and H.G. Sperling (1977) Chromatic organization of primate cones. Science 196:454-456

Matesic, L E., J. Robinson, and J.E. Dowling (1994) Opsin gene expression in zebrafish (Brachydanio rerio): developmental time course. Invest. Ophthal. Vis. Sci. Suppl. 35:1728.

McDevitt, D.S., S.K. Brahma, and J.C. Jeanny (1993) Embryonic appearance of rod opsin in the urodele amphibian eye. Roux's Arch. Dev. Biol. 203:164-168.

Müller, H. (1952) Bau und Wachstum der Netzhaut des Guppy (Lebistes reticulatus). Zool. Jb. 63:275-324.

Nawrocki, L.W. (1985) Development of the neural retina in the zebrafish, Brachydanio rerio. Ph.D. Dissertation, University of Oregon.

Nawrocki, L., R. BreMiller, G. Streisinger, and M. Kaplan (1985) Larval and adult visual pigments of the zebrafish, Brachydanio rerio. Vision Res. $25: 1569-1576$.

Packer, O., A.E. Hendrickson, and C.A. Curcio (1990) Developmental redistribution of photoreceptors across the Macaca nemestrina (pigtail macaque) retina. J. Comp. Neurol. 298:472-493.

Püschel, A.W., P. Gruss, and M. Westerfield (1992) Sequence and expression pattern of pax 6 are highly conserved between zebrafish and mice. Development 114:643-651.

Raymond, P.A. (1985a) The unique origin of rod photoreceptors in the teleost retina. Trends Neurosci. 8:12-17.

Raymond, P.A. (1985b) Cytodifferentiation of photoreceptors in larval goldfish: delayed maturation of rods. J. Comp. Neurol. 236:90-105.

Raymond, P.A. (1986) Movement of retinal terminals in goldfish optic tectum predicted by analysis of neuronal proliferation. J. Neurosci. $6: 2479-2488$.

Raymond, P.A. (1991). Cell determination and positional cues in the teleost retina: development of photoreceptors and horizontal cells. In D.M.K.
Lam and C. Shatz (eds): Development of the Visual System. Cambridge, MA: MIT Press, pp. 59-78.

Raymond, P.A. (1995) Development and morphological organization of photoreceptors. In M.B.A. Djamgoz, S. Archer, and S. Vallegra (eds): Neurobiology and Clinical Aspects of the Outer Retina. London: Chapman and Hall, pp. 1-23.

Raymond, P.A., and P.K. Rivlin (1987) Germinal cells in the goldfish retina that produce rod photoreceptors. Dev. Biol. 122:120-138.

Raymond, P.A., and L.K. Barthel (1994) Origin of rod photoreceptors in zebrafish embryos. Invest. Ophthalmol. Vis. Sci. Suppl. 35:1727.

Raymond, P.A., L.K. Barthel, M.E. Rounsifer, S.A. Sullivan, and J.K. Knight (1993a) Expression of rod and cone visual pigments in goldfish and zebrafish: A rhodopsin-like gene is expressed in cones. Neuron 10:116174.

Raymond, P.A., L.K. Barthel, and G.A. Curran (1993b) Recruitment of photoreceptors in zebrafish embryos. Mol. Biol. Cell 4:374a.

Reh, T.A. (1992) Generation of neuronal diversity in the vertebrate retina. In M. Shankland and E.R. Macagno (eds): Determinants of Neuronal Identity. New York: Academic Press, pp. 433-467.

Robinson, J., E.A. Schmitt, F.I. Hárosi, R.J. Reece, and J.E. Dowling (1993) Zebrafish ultraviolet visual pigment: Absorption spectrum, sequence, and localization. Proc. Natl. Acad. Sci. U.S.A. 90:6009-6012.

Robinson, J., G.B. Grant, and J.E. Dowling (1994) Opsin gene expression in zebrafish (Brachydanio rerio). Soc. Neurosci. Abstr. 20:1322.

Robinson, S.R. (1987) Ontogeny of the area centralis in the cat. J. Comp. Neurol. 255:50-67.

Reece, R J., J. Robinson, and J.E. Dowling (1994) Zebrafish (Brachydanio rerio) visual pigments: sequence and location. Invest. Ophthal. Vis. Sci. Suppl. 35:,1710.

Saha, M.S., and R.M. Grainger (1993) Early opsin expression in Xenopus embryos precedes photoreceptor differentiation. Mol. Brain Res. 17:307318.

Saha, M.S., M. Servetnick, and R.M. Grainger (1992) Vertebrate eye development. Curr. Opin. Genet. Dev, 2,582-588.

Schmitt, E.A., and J.E. Dowling (1994) Early eye morphogenesis in the zebrafish, Brachydanio rerio. J. Comp. Neurol. 342:1-11.

Stenkamp, D.L., and P.A. Raymond (1994) Photoreceptor patterning in embryonic and adult goldfish retina. Mol. Biol. Cell 5:230a.

Stone, J. (1988) The origins of the cells of vertebrate retina. Prog. Retinal Res. $7: 1-19$.

Szél, A., T. Diamantstein, and P. Röhlich (1988) Identification of the blue-sensitive cones in the mmmalian retina by anti-visual pigment antibody. J. Comp. Neurol. 273: 593-602.

Szél, A., P. Röhlich, K. Mieziewska, G. Aguirre, and T. Van Veen (1993) Spatial and temporal differences between the expression of short- and middle-wave sensitive cone pigments in the mouse retina: A developmental study. J. Comp. Neurol. 331:564-577.

Szél, A., T. Van Veen, and P. Röhlich (1994) Retinal cone differentiation. Nature 370: 336

Uehara, F., N. Ohba, Y. Nakashima, T. Yanagita, M. Ozawa, and T. Muramatsu (1993) A fixative suitable for in situ hybridization histochemistry. J. Histochem. Cytochem. 41:947-953.

Usukura, J., W. Khoo, T. Abe, T. Shinohara, and M. Breitman (1993) Abnormal development of cone cells in transgenic mice ablated of rod photoreceptor cells. Ann. N.Y. Acad. Sci. 707:550-553.

Usukura, J., W. Khoo, T. Abe, M..L. Breitman, and T. Shinohara (1994) Cone cells fail to develop normally in transgenic mice showing ablation of rod photoreceptor cells. Cell Tissue Res. 275:79-90.

Wässle, H, and H.J. Reimann (1978) The mosaic of nerve cells in the mammalian retina. Proc. Roy. Soc. Lond. [Biol.] 200:441-461.

Westerfield, M. (1993) The Zebrafish Book: A Guide for the Laboratory Use of Zebrafish (Brachydanio rerio). Eugene, OR: University of Oregon Press.

Wikler, K.C., and P. Rakic (1991) Relation of an array of early-differentiating cones to the photoreceptor mosaic in the primate retina. Nature 351:397400.

Wikler, K.C., and P. Rakic (1994) An array of early differentiating cones precedes the emergence of the photoreceptor mosaic in the fetal monkey retina. Proc. Natl. Acad. Sci. U.S.A. 91:6534-6538.

Wikler, K.C., R.W. Williams, and P. Rakic (1990) Photoreceptor mosaic number and distribution of rods and cones in the rhesus monkey retina J. Comp. Neurol. 297:499-508.

Wong, R.O., and A. Hughes (1987) Role of cell death in the topogenesis of neuronal distributions in the developing cat retinal ganglion cell layer. I. Comp. Neurol, 262:496-511. 